Astrophysics and Space Science manuscript No.

(will be inserted by the editor)

Jeremy S. Heyl

\title{
QED can explain the non-thermal emission from SGRs and AXPs : Variability
}

Received: date / Accepted: date

\begin{abstract}
Owing to effects arising from quantum electrodynamics (QED), magnetohydrodynamical fast modes of sufficient strength will break down to form electron' positron pairs while traversing the magnetospheres of strongly magnetised neutron stars. The bulk of the energy of the fast mode fuels the development of an electronpositron fireball. However, a small, but potentially observable, fraction of the energy $\left(\sim 10^{33}\right.$ ergs $)$ can generate a non-thermal distribution of electrons and positrons far from the star. This paper examines the cooling and radiative output of these particles. Small-scale waves may produce only the non-thermal emission. The properties of this non-thermal emission in the absence of a fireball match those of the quiescent, non-thermal radiation recently observed non-thermal emission from several anomalous X-ray pulsars and soft-gamma repeaters. Initial estimates of the emission as a function of angle indicate that the non-thermal emission should be beamed ' and therefore one would expect this emission to be pulsed as well. According to this model the pulsation of the nonthermal emission should be between 90 and 180 degrees out of phase from the thermal emission from the stellar surface.
\end{abstract}

Keywords gamma-rays: observations · pulsars: individual SGR 1806-20, AXP 4U 0142+61, AXP 1E 1841-045 . radiation mechanisms: non-thermal

PACS 97.60.Jd $\cdot$ 98.70.Rz $\cdot$ 12.20.Ds $\cdot 52.35 . \mathrm{Tc}$

\section{Introduction}

Simply put magnetars are neutron stars whose magnetic fields dominate their emission, evolution and manifes-

The Natural Sciences and Engineering Research Council of Canada, Canadian Foundation for Innovation and the British Columbia Knowledge Development Fund supported this work. J.S.H. is a Canada Research Chair.

Jeremy S Heyl

University of British Columbia 6224 Agricultural Road, Vancouver, BC, Canada V6T 1Z1 E-mail: heyl@phas.ubc.ca tations. In the late 1970s and early 1980s, a fleet of sensitive detectors of high-energy radiation uncovered two new phenomena, the soft-gamma repeater and the anomalous x-ray pulsar. Strongly magnetized neutron stars provide the most compelling model for both types of object, and observations over the past few years indicate that these phenomena are two manifestations of the same type of object. Soft-gamma repeaters exhibit quiescent emission similar to that of anomalous x-ray pulsars (e.g Rothschild et al. 1994; Murakami et al. 1994; Hurlev et al. 1996, 1999), and anomalous x-ray pulsars sometimes burst (Gavriil et al. 2002; Kaspi et al. 2003). What makes magnetars a hot topic of research is the rich variety of physical phenomena that strong magnetic fields exhibit.

This article will focus on the quiescent emission from these interesting objects rather than the bursts themselves (The reader may wish to refer to the seminal work of Thompson \& Duncan (1995) for details of the burst but may also want to look at Hevl \& Hernquist (2005b) for an alternative). Furthermore, the article will concentrate on a possible model for the recently detected nonthermal emission from these objects.

In earlier work, Lars Hernquist and I analysed wave propagation through fields exceeding the quantum critical value $B_{\mathrm{QED}} \equiv m^{2} c^{3} / e \hbar \approx 4.4 \times 10^{13} \mathrm{G}$, and demonstrated circumstances under which electromagnetic (Hevl \& Hernquist 1998b) and some MHD waves, particularly fast modes (Hevl \& Hernquist 1999) evolve in a non-linear manner and eventually exhibit discontinuities similar to hydrodynamic shocks, owing to vacuum polarisation from quantum electrodynamics (QED). In Paper I (Hevl \& Hernquist 2005b), we developed a theory to account for bursts from SGRs and AXPs based on "fast-mode breakdown," in which the wave energy is dissipated into electronpositron pairs when the scale of these discontinuities becomes comparable to an electron Compton wavelength. We showed that, under appropriate conditions, an extended, optically thick pair-plasma fireball would result, radiating primarily in hard X-rays and soft $\gamma$-rays. In $\mathrm{Pa}$ per II (Hevl \& Hernquist 2005a) we developed the the- 
ory of the non-thermal emission from the fast-mode cascade under a series of assumptions and approximations. In particular, MHD fast modes of insufficient amplitude to generate an optically thick fireball will still dissipate through pair-production, seeding non-thermal emission. Hevl \& Hernquist (2005b) estimates the Thomson optical depth through the pair plasma created by a passing fast mode (their Fig. 3). If this optical depth exceeds unity, one would expect a fireball to form.

In what follows, I extend our previous investigation of fast-mode breakdown to estimate the spectrum of nonthermal emission expected outside the region containing an optically thick fireball with particular emphasis on the assumptions made in Paper II and on the angular dependence of this emission.

\section{The Observed Non-Thermal Emission}

What would be the typical flux of these small-scale fast modes? Thompson \& Duncan (1996) and Hevl \& Kulkarni (1998) have argued that the quiescent emission of SGR and AXP neutron stars may be powered by the decay of the magnetic field. The quiescent thermal emission may only be a small fraction of the total energy released by the decay of the magnetic field. Recent observations of SGRs and AXPs indicate that the thermal radiation may indeed be just the tip of the iceberg (Molkov et al. 2004; Mereghetti et al. 2004; Kuiper et al. 2004); therefore, following the discussion of Paper II, the amplitude of the thermal and non-thermal radiation are taken to be comparable, and the pair cascade operates beyond a certain radius from the star or equivalently below a certain magnetic field strength $\left(B_{\max }\right)$. This model has two parameters, $B_{\max }$ determines the position of the two breaks in the spectrum and the total normalisation. The process of fast-mode breakdown according to Paper II predicts a particular relationship between the location of the two breaks in the spectrum and particular slopes between and beyond the breaks.

Figure 11 shows the observed broad-band spectrum of several AXPs and SGRs. To compare the spectra the more distant objects (AXP 1E 1841-045 and SGR 180620) whose hard X-ray emission was discovered with INTEGRAL have been placed at the distance of 4U 0142+61 whose optical emission (Hulleman et al. 2000) is very likely to be nonthermal (Ozel 2004). The observed spectra depart from a power law for $E<1 \mathrm{keV}$ because in this region the thermal radiation from the neutron star begins to dominate the non-thermal component.

Because the location of the two breaks in the spectrum both depend on the strength of the magnetic field at the inner edge of the breakdown region, the presence of extensive nonthermal optical emission indicates that the non-thermal hard X-ray emission should peak at about $30 \mathrm{MeV}$, a factor of two hundred beyond the observed spectrum. The best limits in this energy range are pro-

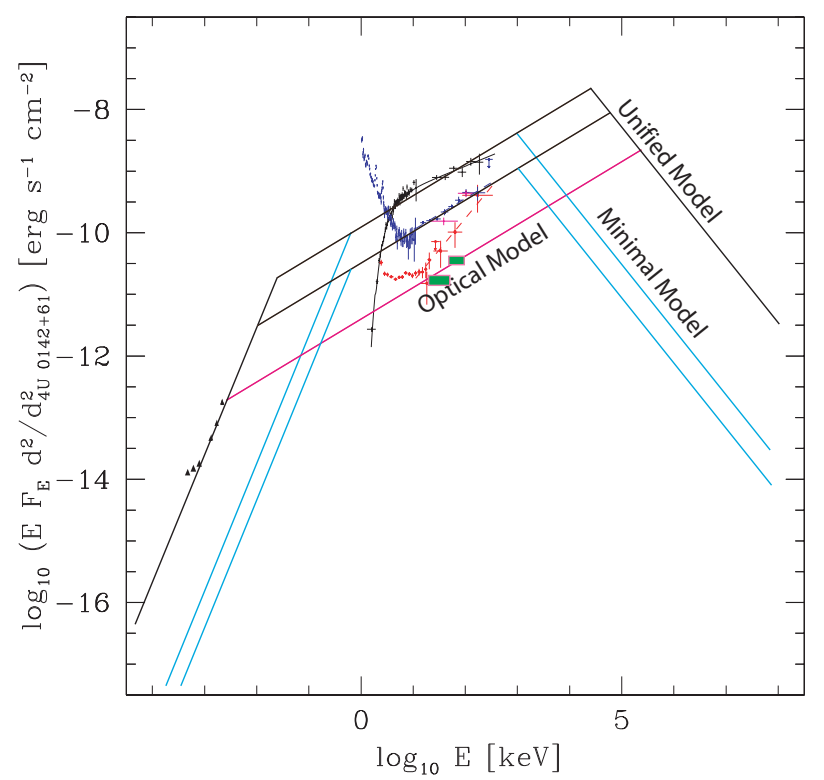

Fig. 1 The spectrum produced by fast-mode breakdown is superimposed over the observed thermal and non-thermal emission from several AXPs and SGRs for models that fit either the optical or INTEGRAL data solely and one that fits both sets of data. The unabsorbed optical data are from Hulleman et al. (2000) via Ozel (2004) for AXP 4U 0142+61. The uppermost black symbols are the hard X-ray band are from Molkov et al. (2004) for SGR 1806-20. Mereghetti et al. (2004) obtained similar results for the SGR. The middle sets of points in the hard X-ray data (blue is total flux and red is pulsed flux) are from Kuiper et al. (2004) for AXP $1 \mathrm{E}$ 1841-045. The green squares plot the INTEGRAL data reported by den Hartog et al. (2004) for AXP 4U 0142+61. The den Hartog et al. (2004) results are normalised using the observations of the Crab by Jung (1989). We scaled the emission from the three sources by assuming that they all lie at the distance of AXP $4 \mathrm{U} 0142+61$. The assumed distances are $3 \mathrm{kpc}$ for AXP 4U 0142+61 (Hulleman et al. 2000), $7.5 \mathrm{kpc}$ for AXP 1E 1841-045 (Sanbonmatsu \& Helfand 1992) and 15 kpc for SGR 1806-20 (Molkov et al.|2004).

vided by Comptel (Kuiper et al. 2006) and appear to exclude the "optical model" for 4U 0142+61.

This conclusion assumes that the sources SGR 180620 and AXP 1E 1841-045 have a similar optical excess to $4 \mathrm{U} 0142+61$. A more conservative assumption would be that the hard X-ray emission does not extend far beyond the observations from INTEGRAL with spectral breaks at about $1 \mathrm{MeV}$ and $650 \mathrm{eV}$. This situation is somewhat natural. The fast-mode cascade is limited to pairs with sufficient energy to produce photons with $E>1 \mathrm{MeV}$ that can subsequently pair produce. Lower energy electrons simply cool, giving the observed cooling spectrum in the hard X-rays. The total energy in the non-thermal emission is also reduced by a factor of a few. In the context of the fast-mode cascade it is difficult to have $E_{\text {break }}<2 m c^{2}$. This model is denoted as the "Minimal Model" in Fig. 11 because $E_{\text {break }}$ takes 
on the minimal value that makes sense physically; i.e $\approx 1 \mathrm{MeV}$; this is equivalent to assuming that magnetic field is much weaker than the quantum-critical limit in the pair-production region.

The analysis reviewed here from Paper I assumed that the fast-mode was travelling perpendicular to the field and that the amplitude of the wave and the magnetic field where the shock forms are independent. The more detailed analysis that follows shows that the location of shock formation depends on the product of initial amplitude of the wave and its wavenumber, its direction of propagation and the strength of the dipole component of the star's magnetic field. The latter two parameters could potentially be determined independently of the emission spectrum, leaving a single free parameter to describe the emission, the product of initial amplitude of the wave and its wavenumber.

\section{Spectrum}

The previous calculation of the spectrum assumed that the energy dissipated by the wave over a given range of magnetic field strengths is constant and that the dissipation only occurs below a particular magnetic field strength. A direct calculation the evolution of the wave as it travels away from the star relaxes both of these assumptions. This determines that amount of energy dumped into pairs at various magnetic field strengths; however, it does not determine how this energy is finally dissipated. It can be emitted locally and promptly as classical synchrotron radiation or Landau transition radiation or elsewhere as curvature emission.

\subsection{Weak-Field Regime}

If the magnetic field is much weaker than the quantumcritical limit, the spectrum of the cooling pairs at a particular value of the magnetic field is given by

$\frac{d E}{d E_{\gamma}}=\frac{E_{\perp}}{2\left(E_{\mathrm{break}}^{1 / 2}-E_{0}^{1 / 2}\right)} E_{\gamma}^{-1 / 2}$

where $E_{0}=m_{e} c^{2} \xi, E_{\mathrm{break}} \approx m_{e} c^{2} /(40 \xi)$ and $\xi=B / B_{\mathrm{QED}}$ for $B \ll B_{Q E D}$. This assumes that the final generation of pairs emits classical synchrotron radiation as described in Paper II and that only the momentum perpendicular to the magnetic field is dissipated as synchrotron emission.

\subsection{Strong-field Regime}

For $\xi \gtrsim 0.1$ the analysis of the preceding section breaks down. This is signaled by the fact that $E_{0} \gtrsim E_{\text {break }}$, so Eq. (11), for example, does not make sense. In this regime both the processes of pair production and synchrotron emission are strong affected. Additionally photon splitting may play a role. Restricting the results to $\xi \ll 1$ yields the results of Hevl \& Hernquist (2005a).

Specifically in the strong-field regime, neither the primary pairs nor the secondaries can be assumed to have relativistic motion perpendicular to the magnetic field. The shock travels at a velocity (Hevl \& Hernquist 1997b),

$v=\frac{c}{n_{\perp}} \approx c\left[1-\frac{\alpha_{\mathrm{QED}}}{4 \pi} X_{1}\left(\frac{1}{\xi}\right) \sin ^{2} \theta\right]^{-1}$

where the function is defined in Hevl \& Hernquist 1997a and well approximated by the fitting formula

$X_{1}\left(\frac{1}{\xi}\right) \approx-\frac{14}{45} \xi^{2} \frac{1+\frac{6}{5} \xi}{1+\frac{4}{3} \xi+\frac{14}{25} \xi^{2}}$

from Potekhin et al. (2004).

The primary pairs typically have a Lorentz factor of

$\gamma=\left(1-\frac{v^{2}}{c^{2}}\right)^{-1 / 2} \approx \frac{1}{|\xi \sin \theta|}\left(\frac{45 \pi}{7 \alpha_{\mathrm{QED}}} \frac{1+\frac{4}{3} \xi+\frac{14}{25} \xi^{2}}{1+\frac{6}{5} \xi}\right)^{1 / 2}$

The typical Landau level occupied by these primary pairs is

$n \approx \frac{45}{14} \frac{\pi}{\alpha_{\mathrm{QED}}} \frac{1}{\xi^{3}} \frac{1+\frac{4}{3} \xi+\frac{14}{25} \xi^{2}}{1+\frac{6}{5} \xi}$

independent of angle, so for $\xi>27$ only the ground Landau level is occupied by the primary pairs. Usov \& Melrose (1995) found a limit of $\xi>0.1$ for pairs to be created of pairs in the ground Landau level. The limit found here is quite a bit different simply because the pair cascade discussed here is due to photons that are essentially created above the pair-production threshold in the shock. The direction of the photons is constant relative to the field direction; it is their energy that increases above the pair-production threshold; they carry much momentum perpendicular to the field. On the other hand, Usov \& Melrose (1995) examine the pair production by photons travelling nearly parallel to the magnetic field. The momentum perpendicular to the field is small so the ground Landau level is preferred at much lower fields.

The Landau level of the secondaries is a factor of sixteen smaller, so for $\xi>8$ only the ground level is occupied by the secondary pairs. In these cases there is no prompt emission at all from the pairs. The energy of the initial photon goes into the rest mass of the pairs and their motion along the magnetic field. If $n$ is not large, the emission is better described by cyclotron emission than by synchrotron. The emission is not cutoff by considerations of the total pair-production optical depth as in the weak-field case (Hevl \& Hernquist 2005a) but by the Landau levels of the pairs produced in the cascade. In the weak-field limit, Hevl \& Hernquist (2005a) found that the energy of the final pairs in the cascade was a factor of 1,700 lower than the primaries, so for $\xi>0.08$ these final pairs occupy the ground Landau level and 
the synchrotron analysis of the cascade emission must be modified; the limiting field for the final pairs in a cascade to be in ground Landau level is similar to that found by Usov \& Melrose (1995) for the pulsar cascade. Comparing the values of $E_{0}$ and $E_{\text {break }}$ yields the same limiting field.

\subsection{The Total Spectrum}

The analysis of the pair cascade in the strong-field limit is beyond the scope of this small contribution. Instead I shall focus on the regime where the entire cascade is in the weak-field limit. This may occur for a very strong wave propagating near a weakly magnetised star or for a relatively weak wave travelling near a strongly magnetised star.

In an arbitrary magnetic field, the evolution of a fast mode can be parametrized by the integral of the "opacity to shocking"

$\kappa=\frac{k \sin \theta}{2} \frac{b}{B} \frac{e^{2}}{h c}\left[\sin ^{2} \theta \kappa_{1}+\left(4-3 \sin ^{2} \theta\right) \kappa_{2}\right]$

along the path of the wave. In this expression, $b$ is the amplitude of the magnetic field of the fast mode, $B$ is the amplitude of the local magnetic field, $\theta$ is the angle between the direction of propagation and the magnetic field and $k$ is the wavenumber of the fast mode.

Although this quantity is not strictly an opacity in the sense of radiative transfer, it does characterize the evolution of the wave in an analogous way. In particular, the shape of the wave depends on the optical depth for shocking

$\tau=\int \kappa d l$.

The shock forms at $\tau=1$, and the power carried by the wave decreases as $\tau$ increases above unity.

The quantity in brackets is the generalization of the function $\xi F(\xi)$ where $F(\xi)$ was defined by Hevl \& Hernquis (1998b). The two terms are

$\kappa_{1}=-\xi^{-3} X_{0}^{(3)}\left(\frac{1}{\xi}\right)$
$\kappa_{2}=X_{0}^{(2)}\left(\frac{1}{\xi}\right) \xi^{-2}-X_{0}^{(1)}\left(\frac{1}{\xi}\right) \xi^{-1}$

Fig. 2 and Fig. 3 depict the opacity as a function of angle and magnetic field strength. For weak fields the opacity is proportional to $\sin \theta$; while in strong fields, it is proportional to $\sin \theta\left(2-\sin ^{2} \theta\right)$.

The definition of $X_{0}(x)$ from Hevl \& Hernquist (1997a) yields

$$
\begin{aligned}
\kappa_{1} & =\frac{2}{3}+\frac{1}{\xi}+\frac{1}{\xi^{2}}-\frac{1}{2 \xi^{3}} \Psi^{(1)}\left(\frac{1}{2 \xi}\right) \\
& \approx \frac{8}{15} \xi^{2}-\frac{32}{21} \xi^{4}+\frac{128}{15} \xi^{6}-\frac{2560}{33} \xi^{8}+\mathcal{O}\left(\xi^{10}\right)
\end{aligned}
$$

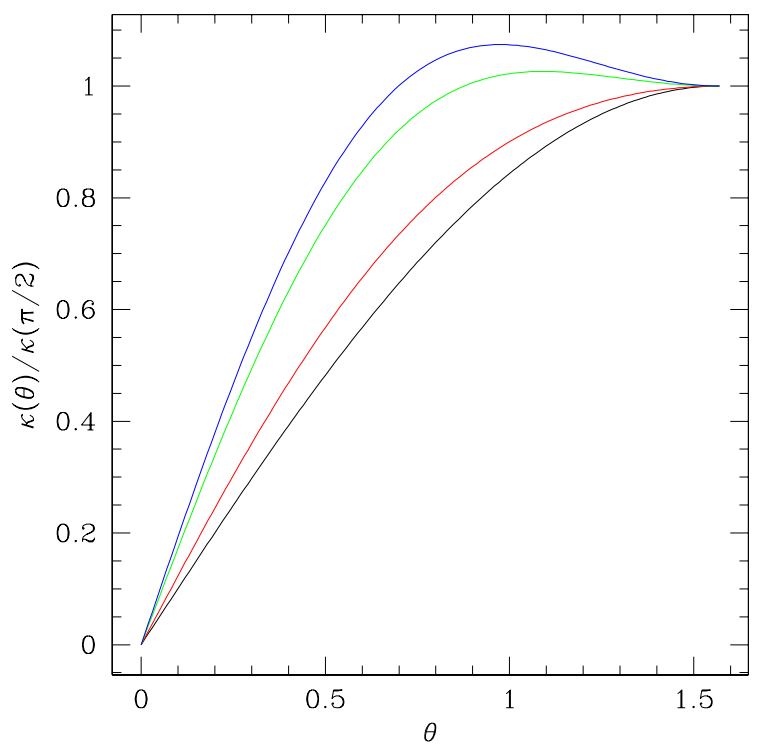

Fig. 2 The opacity as a function of angle at various magnetic field strengths. The curves from bottom to top are for $\xi=0.1,1,10$ and 100 . For weak fields, the opacity peaks at $\theta=\pi / 2$. For strong fields the opacity peaks at $\theta=\cos ^{-1}(1 / \sqrt{3}) \approx 55^{\circ}$.

$$
\begin{aligned}
& \approx \frac{2}{3}-\frac{1}{\xi}+\frac{1}{\xi^{2}}-\frac{\pi^{2}}{12} \frac{1}{\xi^{3}}+\frac{\zeta(3)}{2} \frac{1}{\xi^{4}}+\mathcal{O}\left(\frac{1}{\xi^{5}}\right) \\
\kappa_{2} & =\frac{2}{3}+\frac{\ln (4 \pi \xi)-2 \ln \Gamma\left(\frac{1}{2 \xi}\right)+1}{\xi}+\frac{\Psi\left(\frac{1}{2 \xi}\right)-1}{\xi^{2}} \\
& \approx \frac{8}{45} \xi^{2}-\frac{32}{105} \xi^{4}+\frac{128}{105} \xi^{6}-\frac{2560}{297} \xi^{8}+\mathcal{O}\left(\xi^{10}\right) \\
& \approx \frac{2}{3}+\frac{\ln \left(\frac{\pi}{\xi}\right)}{\xi}-\frac{1}{\xi^{2}}+\frac{\pi^{2}}{24} \frac{1}{\xi^{3}}-\frac{\zeta(3)}{6} \frac{1}{\xi^{4}}+\mathcal{O}\left(\frac{1}{\xi^{5}}\right)
\end{aligned}
$$

where $\Psi(x) \equiv d \ln \Gamma / d x, \Psi^{(1)}(x)=d \Psi / d x$ and $\zeta(3) \approx$ 1.202. Both the opacity and the energy available for stompt synchrotron emission drop as the angle of propagation departs from perpendicular.

The total optical depth is simply the integral of the opacity over the path of the wave. If the wave is spherical, the value of $b$ decreases as $1 / r$ as the wave travels away from the star. For simplicity the angle of the wave propagation with respect to the field is held constant; this is appropriate for waves travelling radially and holds approximately for waves that have travelled several stellar radii from the surface. The dissipation of the energy of the wave is given by Eq. 72 in Hevl \& Hernquist (1998b) and Fig. 2 in Hevl \& Hernauist (2005b).

Summing over all of the magnetic fields where the dissipation occurs yields

$\frac{d E_{\perp}}{d B}=-(1-\cos \theta) \frac{d P}{d B}$

where $P$ is the power remaining in the wave and $\theta$ is the angle that the direction of propagation of the wave 


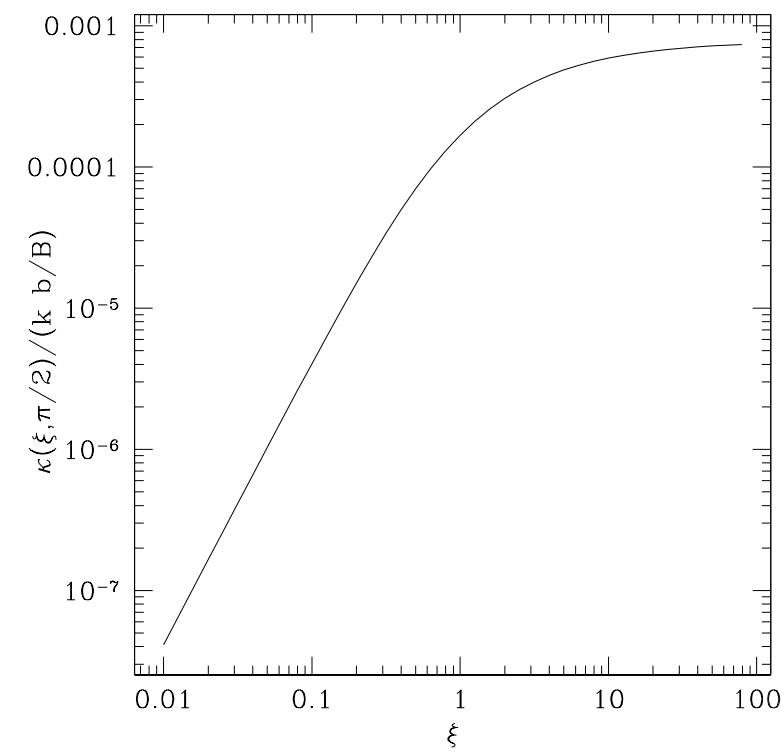

Fig. 3 The opacity as a function of the strength of the field at $\theta=\pi / 2$ in units of $k$ with $b=B$. The reciprocal of the ordinate is the number of wavelengths that a wave of amplitude equal to the background field will travel before forming a shock.

makes with the magnetic field. The angular dependence here comes from the assumption that only the component of the momentum perpendicular to the field yields prompt and local emission. Furthermore, this equation also assumes that the emitting pairs are produced in a weak-field region; they occupy high Landau levels, so the classical treatment of synchrotron emission holds.

For large distances from the neutron star, $d P / d B$ is a constant and the fields are weak; this in combination with the results of $\S 3.1$ yields the spectra given in Hevl \& Hernquist (2005a) and shown in Fig. 4 where the opacity depends on angle as in Eq. (6). The vertical axis gives the fraction of the energy of the initial wave that is dissipated over a factor of $e \approx 2.7$ in energy. For the weak-field treatment to be accurate, both the frequency and the amplitude of the wave must be sufficiently small to delay the formation of the shock until the weak-field regime. This results in a wave that only dissipates a small fraction of its energy into pairs.

In this case the total optical depth to shocking is only slightly greater than unity $\left(\tau_{90}=1.08\right)$ so the spectrum depends strongly on the angle of propagation as depicted in Fig. (4 The radiation is emitted only if $\tau>1$, so the radiation is emitted only for $\sin \theta \tau_{90}>1$ if the bulk of the opacity was in weak magnetic fields, and $\sin \theta\left(2-\sin ^{2} \theta\right) \tau_{90}>1$ if it was in weak magnetic fields. Because the star in this case has $\xi=30$ but the shock does not form until $\xi<0.1$, one would expect the cutoff to lie between these extremes. These two estimates yield cutoff angles of $67^{\circ}$ and $33^{\circ}$. The detailed calculations

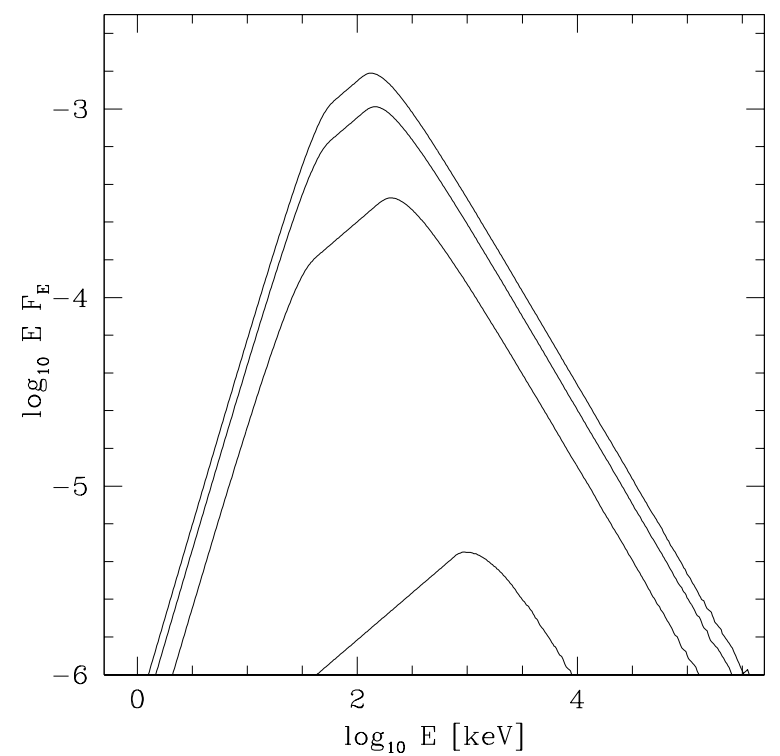

Fig. 4 Total spectrum for strong-field neutron star $\left(B_{\mathrm{NS}}=\right.$ $\left.30 B_{\mathrm{QED}}\right)$ as a function of angle. The initial wave has $\lambda \sim$ $100 \mathrm{~m}$ and $b \sim 2 B_{\mathrm{QED}}$. The curves from shortest to tallest are for $\theta=60^{\circ}, 70^{\circ}, 80^{\circ}$ and $90^{\circ}$.

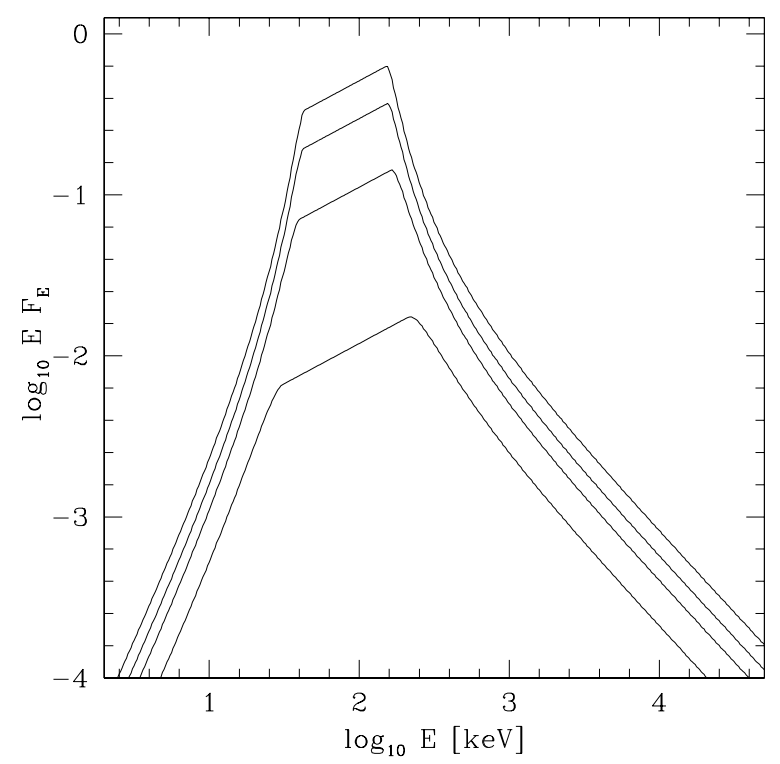

Fig. 5 Total spectrum for weak-field neutron star $\left(B_{\mathrm{NS}}=\right.$ $\left.0.1 B_{\mathrm{QED}}\right)$ as a function of angle. The initial wave has $\lambda \sim 20 \mu$ and $b \sim 0.01 B_{\mathrm{QED}}$. The curves from shortest to tallest are for $\theta=22.5^{\circ}, 45^{\circ}, 67.5^{\circ}$ and $90^{\circ}$.

find that the emission cuts off within about $59^{\circ}$ of the field direction.

If the field at the surface of the star is less than about one-tenth of $B_{\mathrm{QED}}$, the discussion of $\S 3.1$ apply, regardless of where the shock forms, yielding the results of Fig. 5 
In this case the shock may form relatively close to the star and a large fraction of the initial energy in the wave is dissipated over a narrow range of magnetic field strengths where the shock forms. The bulk of the energy may be released in the regime where $d P / d B$ is not constant so the characteristic spectrum discussed in Hevl \& Hernquist (2005a) does not emerge. For large angles with respect to the magnetic field, the shock forms further from the star and the bulk of the energy dissipates in the constant- $d P / d B$ regime. Again the emission cuts off beyond a critical angle. Here $\tau_{90}=6.82522$ yielding a cutoff angle of about $8^{\circ}$. Because the optical depth in the perpendicular direction is so large, the bulk of the angular dependence in the emission comes not from the opacity but from the $(1-\cos \theta)$ factor in Eq. 16]

\section{Comparison with Observations}

The model outlined in the preceding sections yields at least one important prediction. The hard x-ray emission if it is produced by fast-mode breakdown will be largest where the line of sight to the pulsar is perpendicular to the local magnetic field. This happens when the equatorial regions of the pulsar face the observer. On the other hand, the thermal emission from the pulsar is expected to be strongest when the polar regions face the observer (e.g. Hevl \& Hernquist 1998a); consequently the hard and soft x-ray emission should be between 90 and 180 degrees out of phase relative to each other.

If the magnetic field of the neutron star consists of a dipole whose dipole moment makes an angle $\beta$ with the spin axis. Furthermore, the line of sight makes an angle $\alpha$ with the spin axis. If $\alpha+\beta \geq 90^{\circ}$, the hard emission peaks at a phase

$\phi=\arccos (-\cot \alpha \cot \beta)$.

relative to the main pulse of soft emission (for $\alpha+\beta>90^{\circ}$ there can be a second pulse of soft emission from the second polar region). Because $\alpha$ and $\beta$ lie between zero and ninety degrees, their cotangents are positive, so $\phi$ must lie between ninety and one hundred eighty degrees. On the other hand if $\alpha+\beta<90^{\circ}$, the hard emission peaks $180^{\circ}$ out of phase from the soft emission.

Kuiper et al. (2006) observed the x-ray emission from several anomalous x-ray pulsars from 0.5 to $300 \mathrm{keV}$. The AXP 1RXS J1708-4009 exhibits variability similar to that described here. In the $1.3-3.9 \mathrm{keV}$-band the emission peaks around phase 0.25 ; while above $8 \mathrm{keV}$ the emission peaks around phase 0.55 (90 degrees away). The peak remains at phase 0.55 to the highest energies measured about $300 \mathrm{keV}$.

The AXP 4U 0142+61 shows a similar effect albeit less dramatically. At the lower energies that Kuiper et al. (2006) examined $(0.5-1.7 \mathrm{keV})$, the pulse profile is double peaked with two peaks about $160^{\circ}$ out of phase. As the energy of the radiation increases to $50 \mathrm{keV}$, the second peak increases in amplitude and the first peak practically vanishes. The AXP $1 \mathrm{E} 2259+586$ shows similar trends but the variability is only detectable up to about $25 \mathrm{keV}$. On the other hand the pulse profile of AXP 1E 1841-045 does not change much from $2.1 \mathrm{keV}$ to $100 \mathrm{keV}$. This might mean that this object is an exception or that the soft thermal emission only begins to dominate at lower energies.

\section{Discussion}

This paper has examined the angular dependence of the non-thermal emission from AXPs and SGRs in the weakfield regime. It is a natural extension of the model for bursts and non-thermal emission presented in Papers I and II. When a dislocation of the surface of the neutron star is sufficiently large, the resulting fast modes will produce sufficient pairs to make the inner magnetosphere of the neutron star opaque to x-rays, generating a fireball. Some small but observable fraction of the energy initially in the fast modes is dissipated outside the opaque region yielding a characteristic fast-mode breakdown spectrum. A second possibility is that the crust of the neutron star is constantly shifting over small scales, generating fast modes whose breakdown is insufficient to produce a fireball. In this case we would associate the non-thermal radiation with the quiescent thermal radiation from the surface of the star. Both are produced by the quasi-continuous decay of the star's magnetic field.

Observations of the AXPs and SGRs continue to surprise, as do theoretical investigations of ultramagnetised neutron stars. This and the preceding papers have presented a unified model for the thermal burst emission and non-thermal emission from ultramagnetised neutron stars. The model has few underlying assumptions: magnetars produce fast modes sufficient to power the nonthermal emission and, more rarely, the bursts, the magnetic field far from from the star is approximately dipolar and quantum electrodynamics can account for the dynamics of pairs and photons in strong magnetic fields. The model for the non-thermal emission from a particular fast mode depends only on the product of the strength of the wave and its wavenumber, the angle the wave propagates relative to the magnetic field direction and the strength of the dipole component of the field. The intensity of the emission depends strongly on the direction of the initial wave. Waves that travel sufficiently close to the direction of magnetic field do not produce non-thermal emission by this mechanism at all. The emission is therefore beamed perpendicular to the magnetic field lines, so the non-thermal emission should be between ninety and one hundred eighty degrees (depending on the viewing geometry) out of phase relative to the thermal emission which is expected to be strongest along the magnetic field lines. 
Further calculations of the fate of the pairs produced in the strong field regions $(\xi \gtrsim 0.1)$ is needed to understand the spectra produced by fast-mode breakdown in general; however, the beaming of the radiation does appear robust. Further observations can easily verify or falsify this model and potentially provide direct evidence for the ultramagnetised neutron stars that power AXPs and SGRs and the macroscopic manifestations of QED processes that account for their unique attributes.

Acknowledgements Correspondence and requests for materials should be addressed to the author (heyl@phas.ubc.ca). This research has made use of NASA's Astrophysics Data System Bibliographic Services.

\section{References}

den Hartog, P. R., Kuiper, L., Hermsen, W., \& Vink, J. 2004, The Astronomer's Telegram, 293, 1

Gavriil, F. P., Kaspi, V. M., \& Woods, P. M. 2002, Nature, 419,142

Heyl, J. S. \& Hernquist, L. 1997a, Phys. Rev. D, 55, 2449

—. 1997b, Journ. Phys. A, 30, 6485

—. 1998a, MNRAS, 300, 599

—. 1998b, Phys. Rev. D, 58, 043005 (10 pages)

-. 1999, Phys. Rev. D, 59, 045005 (5 pages)

-. 2005a, MNRAS, 362, 777

-. 2005b, ApJ, 618, 463

Heyl, J. S. \& Kulkarni, S. R. 1998, ApJL, 506, 61

Hulleman, F., van Kerkwijk, M. H., \& Kulkarni, S. R. 2000, Nature, 408, 689

Hurley, K., Li, P., Kouveliotou, C., Murakami, T., Ando, M., Strohmayer, T., van Paradijs, J., Vrba, F., Luginbuhl, C., Yoshida, A., \& Smith, I. 1999, ApJL, 510, L111

Hurley, K., Li, P., Vrba, F., Luginbuhl, C., Hartmann, D., Kouveliotou, C., Meegan, C., Fishman, G., Kulkarni, S., Frail, D., Bowyer, S., \& Lampton, M. 1996, ApJL, 463, $\mathrm{L} 13+$

Jung, G. V. 1989, ApJ, 338, 972

Kaspi, V. M., Gavriil, F. P., Woods, P. M., Jensen, J. B., Roberts, M. S. E., \& Chakrabarty, D. 2003, ApJL, 588, L93

Kuiper, L., Hermsen, W., den Hartog, P. R., \& Collmar, W. 2006, ApJ, 645, 556

Kuiper, L., Hermsen, W., \& Mendez, M. 2004, ApJ, 613, 1173

Mereghetti, S., Gotz, D., Mirabel, I. F., \& Hurley, K. 2004, ArXiv Astrophysics e-prints, astro-ph/0411695

Molkov, S., Hurley, K., Sunyaev, R., Shtykovsky, P., \& Revnivtsev, M. 2004, ArXiv Astrophysics e-prints, astro$\mathrm{ph} / 0411696$

Murakami, T., Tanaka, Y., Kulkarni, S. R., Ogasaka, Y., Sonobe, T., Ogawara, Y., Aoki, T., \& Yoshida, A. 1994, Nature, 368, 127

Özel, F. 2004, ArXiv Astrophysics e-prints, astro-ph/0404144

Potekhin, A. Y., Lai, D., Chabrier, G., \& Ho, W. C. G. 2004, ApJ, 612, 1034

Rothschild, R. E., Kulkarni, S. R., \& Lingenfelter, R. E. 1994, Nature, 368, 432

Sanbonmatsu, K. Y. \& Helfand, D. J. 1992, AJ, 104, 2189

Thompson, C. \& Duncan, R. C. 1995, MNRAS, 275, 255

-. 1996, ApJ, 473, 322

Usov, V. V. \& Melrose, D. B. 1995, Australian Journal of Physics, 48, 571 